\title{
Um Mecanismo de Offloading para Redes de Sensores Veiculares
}

\author{
Douglas L. L. Moura ${ }^{1}$, Andre L. L. de Aquino ${ }^{2}$, Antonio A. F. Loureiro ${ }^{1}$ \\ ${ }^{1}$ Departamento de Ciência da Computação \\ Universidade Federal de Minas Gerais (UFMG) \\ Caixa Postal 31270-901 - Belo Horizonte - MG - Brasil \\ ${ }^{2}$ Instituto de Computação - Universidade Federal de Alagoas (UFAL) \\ Caixa Postal 57.072-970 - Maceió - AL - Brasil \\ \{douglas.moura, loureiro\}@dcc.ufmg.br, alla@laccan.ufal.br
}

\begin{abstract}
Vehicular ad hoc network refers to an promise approach that vehicles are equiped with wireless communication devices capable to stablish communication with other vehicles. Each vehicle is capable of collecting, processing and disseminating information about Itself and your enviroment. The acquisition of a large amount of sensing information and the increasing demand for traffic cellular requires mechaninsms to decongest the cellular network. Thus, this work uses a measure of centrality to propose an offloading mechanism in which certain vehicles will collect data from their neighbors and transmit it in the cellular uplink. The experiments were conducted in a real scenario and the results showed a reduction of up to $8.79 \%$ in the number of accesses to the cellular network when compared to another solution in the literature.
\end{abstract}

Resumo. A rede veicular refere-se a uma promissora abordagem de ter veículos equipados com dispositivos de comunicação sem fio capazes de estabelecer comunicação com outros veículos. Cada veículo é capaz de recolher, processar e divulgar informações sobre si e seu ambiente. A aquisição de uma grande quantidade de informações de sensoriamento e a demanda crescente de tráfego exige mecanismos para descongestionar a rede celular. Deste modo, este trabalho utiliza uma medida de centralidade para propor um mecanismo de offloading, no qual determinados veículos irão coletar dados de seus vizinhos e transmiti-los no uplink celular. Os experimentos foram conduzidos em um cenário real e os resultados encontrados mostraram um redução de até $8.79 \%$ no número de acessos à rede celular em relação à outra solução da literatura.

\section{Introdução}

Os conceitos básicos de automação de estradas, isto é, o uso de técnicas de comunicação e controle para tornar o tráfego rodoviário seguro, eficiente e amigável, remetem ao ano de 1939, no qual visionários tentaram prever de que forma seria o sistema de transporte no futuro [Hartenstein and Laberteaux 2010]. Diversos projetos de pesquisa foram realizados desde então, contudo, a tecnologia vigente na época era uma limitação. Na década de 90, com o surgimento do Sistema de Posicionamento Global (GPS) e da rede local sem fio (WLAN), pesquisas no campo da comunicação inter-veicular ganharam um impulso considerável. Posteriormente, em 2004 surge a especificação 802.11p WAVE (Wireless 
Access in Vehicular Environment) como proposta de internacionalização do padrão de comunicação inter-veicular. Além disso, a implantação comercial da última geração de tecnologia de comunicação celular tornou disponível uma infraestrutura de comunicação quase universal, o que propiciou o surgimento de novas tecnologias wireless.

A rede ad hoc veicular (Vehicular Ad hoc Network - VANET) refere-se a uma promissora abordagem de ter veículos equipados com dispositivos de comunicação sem fio, denominados de unidades de bordo (Onboard Units - OBUs), capazes de estabelecer comunicação com outros veículos em seu alcance de transmissão [Campolo et al. 2015]. Através da integração de OBUs com a interface de rede, bem como diferentes tipos de sensores e receptores de GPS, cada veículo é capaz de recolher, processar e divulgar informações sobre si e seu ambiente. Em razão disto, a VANET constitui-se como uma importante fonte de informações para um promissor paradigma de sensoriamento remoto, conhecido como redes de sensores veiculares (do inglês, vehicular sensor network - VSN) [Lee et al. 2006, Wang et al. 2018]. Uma variedade de novos serviços é possível a partir do processamento de informações periodicamente coletadas por veículos, por exemplo, melhorar a segurança nas estradas, gerenciamento de tráfego, navegação inteligente, monitoramento de poluição, vigilância urbana e até mesmo investigações forenses [Bazzi et al. 2015].

Além da comunicação exclusiva entre veículos, denotada como V2V (Vehicleto-Vehicle), os veículos também podem comunicar-se com pontos fixos instalados ao longo das estradas, tais infraestruturas são referidas como unidades de acostamento (Roadside Units - RSUs). Este segundo modo de comunicação é referido como (Vehicle-toInfrastructure). Além de expandir a capacidade de comunicação dos veículos, as RSUs são capazes de conectar os veículos à Internet. Contundo, seu alto custo de implantação torna impraticável montar uma infraestrutura capaz de oferecer cobertura a todos os veículos do cenário. Em contrapartida, com a adesão da telefonia móvel e o crescente aumento no número de celulares com tecnologia de última geração, a rede $5 \mathrm{G}$ configurase como um passo fundamental para a chamada Internet das Coisas (Internet of Things IoT), que busca conectar diferentes tipos dispositivos em uma única rede. Embora este cenário favoreça o surgimento de novas aplicações VANETs baseadas na comunicação celular, o rápido crescimento na demanda de tráfego exige meios de diminuir a sobrecarga na rede celular. Está previsto que o tráfego mensal global de dados móveis será de 49 exabytes até 2021, no qual 20\% do tráfego IP será originado por dispositivos móveis [Forecast 2017].

Trabalhos na literatura buscaram resolver este problema fazendo o offloading dos dados móveis, no qual o conteúdo que seria transmitido originalmente na rede celular é descarregado em uma rede complementar e de baixo custo, como Wi-Fi, 802.11p e LTE Device-to-Device (D2D) [Mao et al. 2016]. O objetivo deste trabalho é apresentar uma solução distribuída para realizar o offloading dos dados VSN que seriam transmitidos através do uplink celular, para tal, a solução baseia-se numa medida de centralidade da teoria e análise de grafos, chamada de closeness centrality [Freeman 1978]. Assim, um algoritmo guloso foi proposto para determinar um subconjunto de veículos transmissores que irão utilizar a comunicação V2V para coletar os dados transmitidos por seus vizinhos e realizar a transferência para a estação base. Os experimentos foram conduzidos a partir de traces de mobilidade reais e os resultados encontrados mostraram uma melhora de até 


\subsection{9\% em relação à outra solução da literatura.}

Este trabalho contribui para a literatura propondo uma solução para um problema atual e de grande relevância para a área de redes de comunicações. A contribuição central deste artigo reside no fato de que muitos trabalhos que abordaram este mesmo problema partem de soluções centralizadas ao passo que a solução aqui proposta é distribuída. Além da ausência de um nó coordenando o offloading, este trabalho considerou o descarregamento de dados em cenários tolerantes a atrasos e com requisito de tempo real. Assim, o serviço proposto permite controlar o tamanho da vizinhança utilizada para descarregar os dados VSN e tornar o processo escalável.

O presente trabalho está estruturado em 6 seções. A Seção 2 apresenta uma revisão da literatura, destacando trabalhos relevantes que abordam tópicos similares à proposta desta pesquisa. A Seção 3 aprofunda o tema sobre redes de sensores veiculares e descreve o problema abordado. A Seção 4 descreve o algoritmo proposto como estratégia para solucionar o problema. A metodologia adotada e os resultados encontrados são mostrados na Seção 5. Por fim, a Seção 6 aborda as considerações finais do artigo bem como possíveis trabalhos futuros.

\section{Trabalhos Relacionados}

A largura de banda nas estações bases é limitada, em razão disto, um grande número de transmissões de pacotes na rede celular pode ser monetariamente caro e ineficiente para atender as demandas dos usuários móveis [Lin et al. 2018]. A partir de uma rede complementar, dispositivos podem se comunicar com outros através de conexões ad hoc, utilizando comunicações como D2D, Bluetooth, WiFi ou 802.11p. A conexões são comumente de alta capacidade e podem ser exploradas por dispositivos móveis para o compartilhamento de conteúdo cujo interesse é de caráter comum.

Muitos trabalhos na literatura consideraram o descarregamento de dados móveis na direção de downlink, no qual as estações transmitem o conteúdo para um veículo e este dissemina para os demais a partir de transmissões broadcast [Mezghani et al. 2016, Salvo et al. 2016, Mao et al. 2016, Dua et al. 2017]. Em [Mao et al. 2016], por exemplo, os autores buscaram expandir capacidade de comunicação utilizando D2D e WiFi para integrar diversos dispositivos e formar uma rede heterogênea.

Há ainda trabalhos que concentraram-se em descarregar os dados em uma direção de uplink, quando uma grande quantidade de informações deve ser disponibilizada na Internet e recebida por um servidor remoto para processamento e análise. Em [Lee and Lee 2013], os autores consideram informações históricas da mobilidade dos veículos para prever WLANs disponíveis e realizar o descarregamento dos dados.

Os autores [Stanica et al. 2013] propuseram três soluções distribuídas para lidarem com o problema de selecionar os nós responsáveis pelo upload dos dados de sensoriamento: algoritmo baseado no grau dos nós da rede, algoritmo baseado no grau com um mecanismo de confirmação e um algoritmo baseado em reserva de tempo. Dentre os três algoritmos, o terceiro obteve os melhores resultados quando a cobertura foi uma exigência. Os resultados obtidos mostraram uma redução de até 95\% no acesso a rede celular para o cenário avaliado, contudo, a necessidade de sincronização dificulta uma possível aplicação prática da solução. 
A teoria dos grafos também foi explorada como forma de capturar propriedades estruturais da rede e determinar como será realizado o offloading [Kolios et al. 2013]. Os autores [Yuan et al. 2016] usaram grafos para modelar a probabilidade de conexões entre veículos. Os resultados encontrados mostraram que foi possível descarregar mais de $70 \%$ dos dados em um cenário com restrições de tempo e espaço.

Em seu trabalho, os autores [Bazzi et al. 2015] buscaram resolver o problema de sobrecarga na rede celular utilizando RSUs para coletar e realizar o upload dos dados de sensoriamento. Embora os resultados obtidos tenham mostrado uma redução completa no acesso à rede celular em cenários de alta densidade veicular, a estratégia proposta exigia um algoritmo para determinar como instalar as RSUs no cenário e um algoritmo para rotear o pacote do veículo de origem até a RSU.

Trabalhos como [Li et al. 2014, Li et al. 2015, Zhu et al. 2017, Nunes et al. 2017] exploraram comunicações oportunistas no offloading de dados tolerantes a atrasos. Certos serviços baseados em VSN exigem um upload em tempo quase real, sendo assim, uma desvantagem desta abordagem é que o descarregamento de conteúdo acarretará em um atraso maior em comparação com a transmissão direta usando redes celulares. Os autores [Lin et al. 2018] apresentaram uma solução chamada CUBI para descarregar os dados da rede $4 \mathrm{G}$ para uma rede WiFi. A solução combina o indicador de qualidade de canal (CQI) para determinar qual rede detém a melhor taxa de entrega e utiliza informações procedentes das estações bases para prevenir sobrecarga na infraestrutura celular.

Este trabalho explora informações de mobilidade veicular para selecionar veículos responsáveis por coletar dados de seus vizinhos e realizar o upload na estação base para que possam ser transmitidos para análise e processamento remoto. A solução proposta pode ser distribuída de modo a realizar a computação nas bordas e tornar o esquema de offloading escalável para operar e realizar o sensoriamento em tempo quase real. Deste modo, os resultados deste trabalho foram avaliados comparando com os resultados encontrados por [Stanica et al. 2013], que utilizou o mesmo cenário e também buscou soluções distribuídas.

\section{Offloading em redes veiculares de sensoriamento}

Este trabalho aborda o offloading de uma grande quantidade de dados de sensoriamento em uma direção de uplink, no qual os dados devem ser transmitidos para um centro de processamento na Internet. As aplicações de sensoriamento podem utilizar informações coletadas em tempo quase real ou com tolerância a atrasos, a solução proposta neste artigo foi elaborada intencionando o offloading em ambos os cenários. Uma vez que o processo de upload de informações exige uma cobertura de Internet quase ubíqua, foi considerado um cenário habilitado com comunicação V2I celular, seja ela 4G ou 5G.

As mensagens beacons são geradas pelos veículos em um curto período de tempo com a finalidade de tornar cada veículo consciente de seu ambiente e prevenir acidentes. São mensagens contendo dados agregados sobre seus vizinhos e também informações acerca do estado do veículo remetente, como posição do veículo, velocidade, direção etc. A Figura 1 mostra um exemplo de um serviço baseado na aquisição de mensagens beacons, que podem ser utilizadas por aplicações, como monitoramento de tráfego, ou protocolos, como roteamento.

A primeira etapa do processo de sensoriamento consiste na coleta dos dados. 
Nesta fase, o veículo escolhido para acessar a rede celular irá coletar os dados de sensoriamento que serão transmitidos para o servidor. Os veículos irão tirar proveito da capacidade de comunicação inter-veicular para disponibilizar seus dados para o veículo retransmissor. Em uma abordagem tradicional cada veículo iria acessar o uplink celular e transmitir seus dados individualmente. Em um esquema de offloading, um subconjunto de veículos será selecionado para agregar dados de seus vizinhos e transmiti-los à estação base.

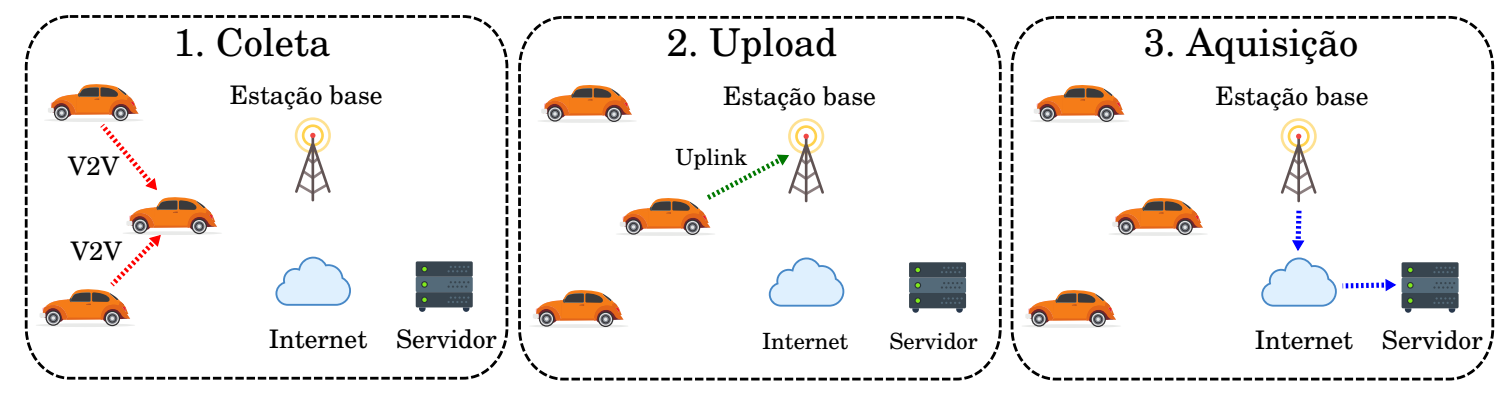

Figura 1. Esquema ilustrando as etapas principais de um offloading.

A segunda etapa consiste de realizar o upload do conteúdo VSN para a estação base no qual o veículo está associado. O veículo escolhido para upload irá fundir os dados recebidos, os dados podem ser empacotados numa mesma mensagem ou transmitidos em mensagens diferentes, porém em uma mesma sessão. A terceira e última etapa trata-se do processo de aquisição dos dados pelo servidor. A estação base irá disponibilizar os dados para o servidor a partir da Internet, no qual os dados de sensoriamento serão processados e analisados remotamente. Estes dados poderão ser utilizados para auxiliar na tomada de decisão de algum serviço ou aplicação, assim, uma grande quantidade de informações é capaz de melhorar a qualidade da amostra e fornecer informações mais representativas do ambiente sensoreado.

Em [Stanica et al. 2013], dentre as soluções elaboradas, os autores propuseram o reservation-based $(R B)$, uma solução distribuída baseada em intervalos de tempo, para realizar o offloading dos dados de sensoriamento. Uma métrica denominada ganho foi definida como forma de medir a qualidade das soluções obtidas pelo RB, ela é definida como a fração de veículos que não utilizaram a infraestrutura celular.

Exigindo o sincronismo de transmissões, o RB é descrito em três etapas:

1. Cada veículo irá escolher aleatoriamente um intervalo de tempo dentre os $N_{s}$ intervalos disponíveis e entrará em estado de contenção;

2. Cada veículo irá aguardar a chegada do seu intervalo de tempo escolhido. Se ele estiver em estado de contenção, o veículo irá transmitir uma mensagem de reserva e entrará em um estado de dominador;

3. Um veículo em estado de contenção que recebeu uma mensagem de reserva de algum vizinho irá cancelar seu estado de contenção e entrará em estado de dominado.

Os veículos que formam o conjunto dos veículos dominadores são aqueles responsáveis por coletar dados dos vizinhos (veículos dominados) e transmitir para a estação base. É difícil garantir que cada veículo transmita em um intervalo de tempo diferente em 
uma implementação real e distribuída do RB. Além disso, um veículo que recebe mais de uma mensagem de reserva que foi transmitida em um mesmo intervalo de tempo pode não ser capaz de decodificar a mensagem.

\section{Multihop baseado em closeness centrality (MHC)}

As redes derivadas de dados reais apresentam estruturas e características que podem ser estudadas através de modelos e métricas [Costa et al. 2007]. A medida de centralidade definida para um veículo é uma métrica que busca classificá-lo de acordo com a sua posição na rede. Assim, a partir da medida de centralidade é possível descobrir quais veículos desempenham maior importância topológica dentro daquela estrutura. A solução descrita neste artigo utiliza o closeness centrality para identificar os veículos que possuem uma maior proximidade com os demais.

O caminho mínimo entre dois vértices em um grafo não-ponderado é definido como a menor sequência de arestas conectando-os. Assim, o closeness centrality $(C)$ de um nó $x$ na rede é uma medida de centralidade definida conforme mostra a Equação 1.

$$
C(x)=\sum_{y \neq x} \frac{1}{d(y, x)}
$$

Onde $d(y, x)$ é a distância do nó $x$ até o nó $y$, ou seja, o tamanho do menor caminho ligando os dois nós. Uma vez que o cálculo das distâncias exige a disseminação de mensagens para a descoberta dos nós, o cálculo de $C(x)$ foi intencionalmente alterado neste trabalho. Aqui, o closeness centrality é calculado apenas para os pares de vértices cujas distâncias são menores ou iguais à $k$.

O multihop baseado em closeness centrality (MHC) parte das comunicações locais estabelecidas por cada veículo para modelar a rede e extrair informações estruturais do grafo. A partir destas informações é possível explorar a mobilidade veicular para determinar a centralidade dos veículos e selecionar aqueles que irão acessar a rede celular. O MHC é descrito em quatro fases:

1. Reconhecimento - Cada veículo utilizará informações trocadas localmente (mensagens beacons, por exemplo) para identificar seus vizinhos imediatos e transmitir estas informações à estação base;

2. Modelagem - A estação base irá unir as informações locais que foram previamente disponibilizadas por cada veículo associado;

3. Seleção - Uma vez que o topologia da rede foi obtida com base nas informações locais, a estação irá utilizar o closeness centrality em uma abordagem gulosa (ver Algoritmo 1) para selecionar quais veículos acessarão o uplink celular;

4. Upload - Nesta fase, um veículo selecionado para acessar o uplink celular irá receber uma mensagem da estação base solicitando o upload. O veículo poderá então transmitir os dados coletados que estão em seu buffer.

A Figura 2 mostra um exemplo de como seriam as soluções construídas pelo MHC. Os vértices coloridos como verde fazem acesso à rede celular, enquanto que os vértices na cor cinza utilizam uma rede complementar para disponibilizar seus dados para outros veículos em seu raio de transmissão. Cada veículo na cor verde está separado dos 
demais veículos verdes com uma distância de pelo menos $k$ saltos. Assim, a Figura 2(a) apresenta o MHC com $k=0$, cuja solução corresponde a um upload tradicional, obtendo um ganho de 0\%. As Figuras 2(b) e 2(c) mostram o MHC considerando o parâmetro $k=1$ com um ganho de $47 \%$ e $k=2$ com um ganho de $60 \%$.

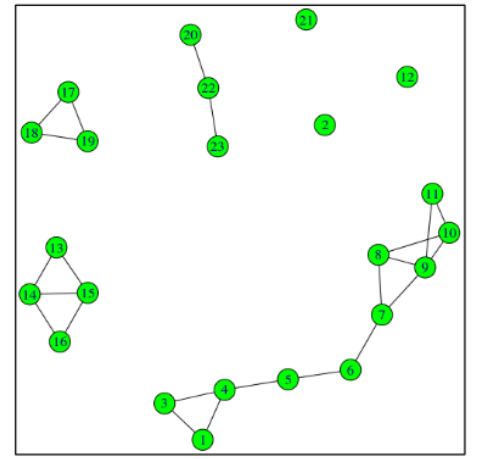

(a) $\mathrm{k}=0$

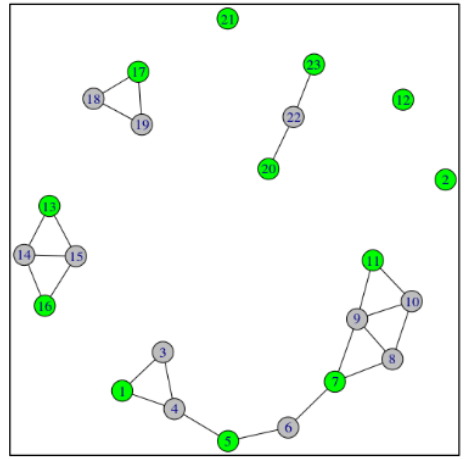

(b) $k=1$

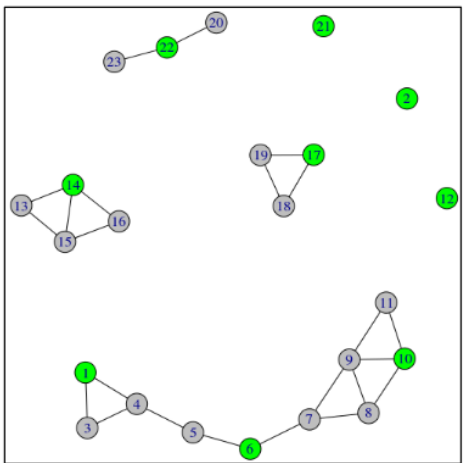

(c) $k=2$

Figura 2. Soluções encontradas em uma rede tomada como exemplo.

O Algoritmo 1 mostra o algoritmo guloso responsável por selecionar os veículos que irão acessar a infraestrutura celular e realizar o upload dos dados coletados. O algoritmo recebe o grafo $G$ que modela a rede e o parâmetro $k$ com o tamanho máximo da distância $d$. Na Linha 2, o algoritmo irá computar todas as distâncias levando $k$ em consideração. Em seguida, o closeness centrality é calculado para todos os nós tomando as distâncias em $d$ (Linha 3). Enquanto existirem vértices em $G$, o vértice com a maior medida de centralidade será removido de $G$ e adicionado no conjunto solução $S$. O veículo em questão irá receber uma mensagem da estação base, notificando-o que o mesmo foi escolhido para coletar dados de seus vizinhos até o k-ésimo nível. Isto assegura que os veículos selecionados para comunicação celular estarão separados por no mínimo $k$ saltos.

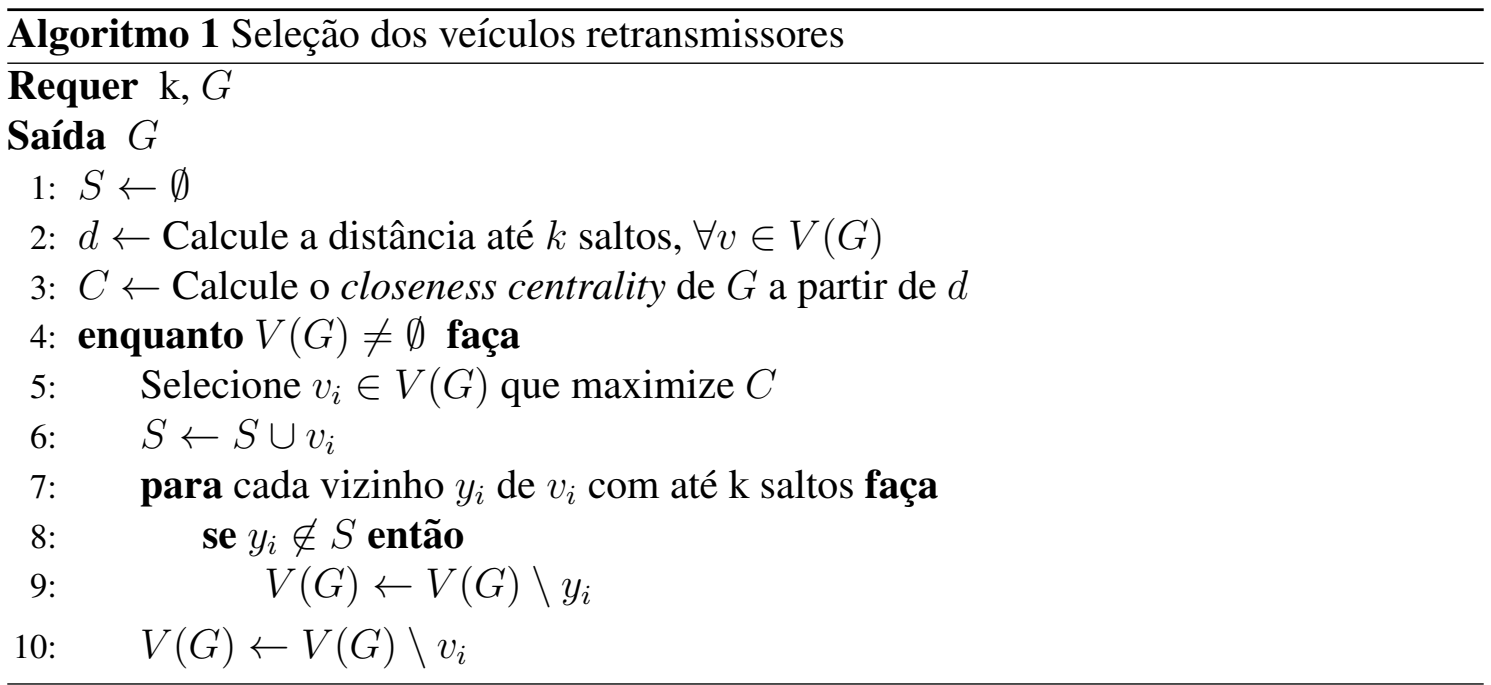

Uma vez que os veículos estão em constante movimento, a estação base precisa atualizar o grafo da rede sempre que for executar o Algoritmo 1. Embora a fase de reconhecimento exija que os veículos tenham que transmitir as identidades de seus vizinhos 
à estação base, estas informações poderiam ser transmitidas no canal de feedback juntamente com outras mensagens de controle. Importante mencionar que o Algoritmo 1 é executado distribuidamente em cada estação base ao passo que cada veículo irá processar a sua própria vizinhança. Embora haja o processamento na estação base, a solução é capaz de explorar a comunicação multihop para diminuir o número de acessos ao uplink e o tráfego no backhaul da rede celular.

\section{Experimentos}

Os experimentos conduzidos neste trabalho pretendem investigar a eficácia da solução proposta em relação aos resultados encontrados pelo RB. Diante disto, este trabalho utilizou um cenário construído a partir do trace de mobilidade da cidade de Colônia, na Alemanha. O dados estão disponíveis pelo projeto TAPASCologne [Uppoor et al. 2014], uma iniciativa do Institute of Transportation Systems (ITS-DLR) no Centro Aeroespacial Alemão. O conjunto de dados original do TAPASCologne cobre uma área de $400 \mathrm{~km}^{2}$ por um período de 24 horas e granularidade de 1 segundo; mais de 700.000 rotas individuais de veículos foram utilizadas.

O layout da área da cidade, Figura 3, foi obtido a partir do OpenStreetMap ${ }^{1}$ (OSM), projeto colaborativo de mapeamento constituído por dados abertos, e a simulação de mobilidade dos veículos foi realizado pelo Simulation of Urban Mobility (SUMO) [Behrisch et al. 2011], simulador de tráfego livre e aberto composto de ferramentas que dão apoio à simulação microscópica e suporte ao formato exportado pelo OSM.

Figura 3. Topologia do cenário utilizado na simulação de mobilidade.

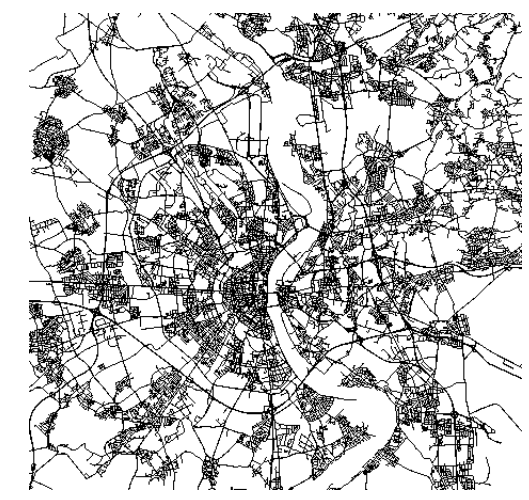

(a) Fragmento do mapa da cidade de Colônia (Alemanha).

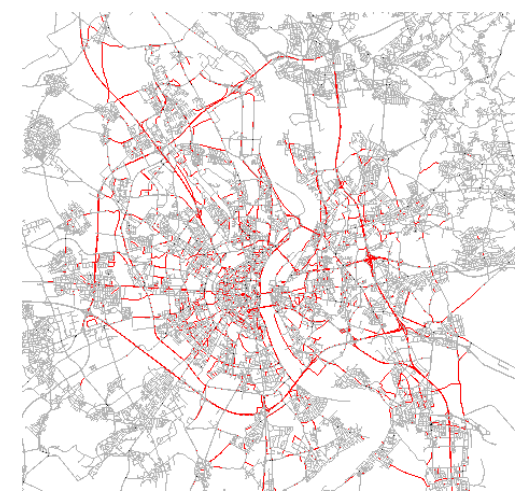

(b) Fluxo de veículos no instante de 7 horas e 30 minutos.

Durante os experimentos, foi considerado que todos os veículos dispunham de capacidade de comunicação V2V, seja ela baseada no padrão 802.11 p ou através de comunicação D2D. Além disso, foi considerado que todos os veículos possuíam cobertura celular ininterrupta, assim, qualquer veículo estaria habilitado com interface de rede celular $(4 \mathrm{G}$ ou $5 \mathrm{G})$ para realizar o upload V2I de seus dados caso necessário. A comunicação V2V foi modelada como um grafo temporal, no qual as ligações entre veículos são estabelecidas conforme o modelo de disco unitário. Os experimentos foram realizados a partir de uma faixa de transmissão de 100 metros, valor comumente empregado em análises

\footnotetext{
${ }^{1}$ Disponível em: https: / /www. openstreetmap.org/
} 


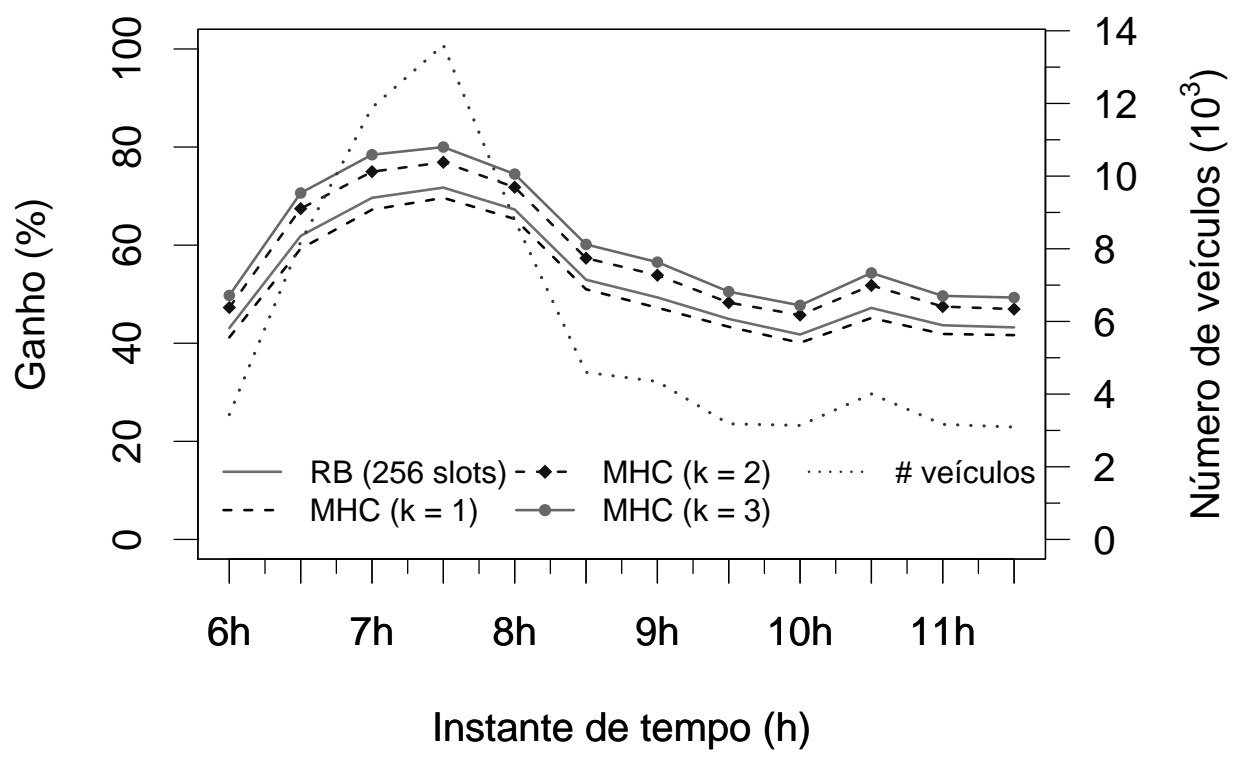

Figura 4. Ganho obtido em um cenário com horário de pico.

experimentais. O grafo foi modelado como não-direcionado, deste modo, pressupõe-se que todos os veículos possuem a mesma potência de transmissão.

A Figura 4 mostra um comparativo entre a solução proposta (MHC) e o RB em um cenário com horário de pico (de $6 \mathrm{~h}$ à $11 \mathrm{~h} 30 \mathrm{~min}$ ). Mais de 13 mil veículos transitaram pelo cenário e realizaram o upload a cada 30 minutos. O número de saltos $(k)$ do $\mathrm{MHC}$ foi variado em 1, 2 e 3. Execuções mostraram que os melhores resultados do RB aparecem com 256 intervalos de tempo, assim, o RB foi avaliado com este valor de parâmetro. Em razão da estocasticidade do $\mathrm{RB}$, o mesmo foi replicado 10 vezes e sua média foi tomada como resultado das execuções.

A partir do gráfico fica evidente como o número de saltos afeta diretamente o ganho obtido pela solução. Em $k=1$, o descarregamento utilizando apenas os vizinhos diretos não foi suficiente para garantir um ganho superior ao RB. O RB foi melhor em todos os instante de tempo, sendo que em 6h30min ele obteve uma diferença de 2.61 pontos percentuais em relação ao ganho do MHC. Este cenário muda quando beneficia-se da comunicação multihop. Em $k=2$, por exemplo, os resultados encontrados por MHC mostraram um aumento de até $5.56 \%$ em relação ao RB quando o offloading foi realizado às 6 h30min. Em contrapartida, o menor ganho foi de 3.33\%, que aconteceu às $9 \mathrm{~h} 30 \mathrm{~min}$.

Em todas as soluções o ganho é proporcional à densidade de veículos no cenário. Isto evidencia-se ao analisar os resultados de $k=3$, que obteve o maior percentual de ganho no descarregamento, um aumento de até $8.79 \%$ em relação ao RB. No instante de $7 \mathrm{~h} 30 \mathrm{~min}$ da manhã, cerca de $80 \%$ dos veículos descarregaram seus dados na rede complementar e não precisaram utilizar da infraestrutura celular. Embora o número de saltos seja capaz de potencializar o descarregamento dos dados a partir de uma exploração mais profunda na vizinhança do veículo, um número maior de saltos exige uma maior troca de mensagens para identificar os veículos retransmissores, e portanto, tem um impacto em sua execução. Ao analisar os resultados é possível averiguar que o benefício obtido por $k$ diminui conforme $k$ cresce. O aumento de $k=2$ em relação à $k=1$ foi de $8.16 \%$ 


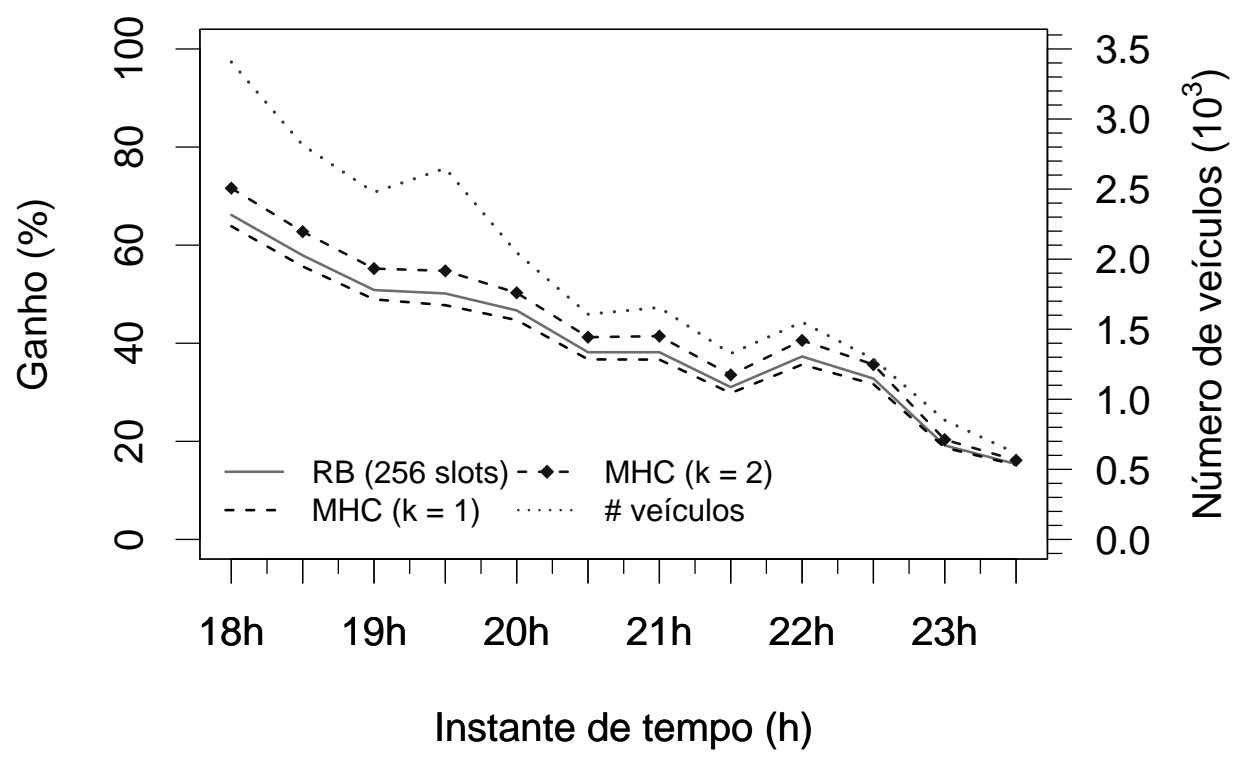

Figura 5. Ganho obtido em um cenário com horário de tráfego moderado e fraco.

enquanto que o aumento de $k=3$ em relação à $k=2$ foi de $3.46 \%$. Isto ocorre em razão da mobilidade dos veículos, pois uma vez que os veículos se movem em diferentes regiões do cenário e em alta velocidade, o grafo da rede sofre com constantes desconexões, o que desfavorece uma transmissão através de um grande número de saltos.

Os experimentos também buscaram analisar o comportamento do MHC diante de um cenário com um trânsito moderado e fraco. A Figura 5 mostra os resultados obtidos em um intervalo de $18 \mathrm{~h}$ às $23 \mathrm{~h} 30 \mathrm{~min}$, com uma granularidade de 30 minutos. Em razão do número menor de veículos, o MHC foi executado com $k=1$ e $k=2$ apenas. Neste cenário é possível perceber uma redução brusca no número de veículos, que em 23h30min chega a contabilizar 615 veículos. Conforme mencionado, o ganho obtido no descarregamento é proporcional ao número de veículos no cenário, assim, os maiores ganhos obtidos foram às $18 \mathrm{~h}$. O MHC conseguiu ganhos de $63.85 \%$ com $k=1$ e $71.61 \%$ com $k=2$, o RB conseguiu $66.15 \%$. Novamente o $k=1$ foi inferior ao $\mathrm{RB}$, contudo, expandido para $k=2$ foi possível um aumento de $5.45 \%$ em relação ao RB. Em contrapartida, em $23 \mathrm{~h} 30 \mathrm{~min}$ o MHC conseguiu ganhos de $15.1 \%$ com $k=1$ e $16.13 \%$ com $k=2$, o RB conseguiu $15.3 \%$.

O ganho obtido pelo offloading decresce em cenários de tráfego moderado e fraco. Isto acontece porque uma vez que o cenário dispõe de pouquíssimos veículos, a rede torna-se esparsa, fragmentada e com desconexões constantes, o que torna difícil beneficiar-se da comunicação inter-veicular para descarregar dados. Neste caso, na ausência de veículos vizinhos em seu raio de transmissão, cabe ao veículo realizar seu próprio upload. O MHC tenta explorar informações sobre a proximidade dos veículos para determinar o veículo que irá coletar dados de seus vizinhos e transmiti-los para a Internet, além disso, o MHC permite definir o nível de exploração dos vizinhos. Assim, valores muito grandes para $k$ é dispendioso para realizar um offloading com alta granularidade de tempo e suas conexões podem ser vulneráveis em um grafo esparso, pois uma vez que o padrão de mobilidade veicular é altamente dinâmico, transmissões poderiam se perder com facilidade diante de um cenário com pouca densidade veicular. 


\section{Conclusões}

Este trabalho apresentou uma solução distribuída para realizar o offloading de dados de sensoriamento em redes veiculares. $\mathrm{O}$ algoritmo proposto segue uma abordagem gulosa e baseia-se numa medida de centralidade como forma de capturar a relação de proximidade entre os veículos e utilizá-la como informação para guiar a construção da solução. Os resultados encontrados mostraram um aumento de até $8.79 \%$ em relação ao RB em um cenário de trânsito intenso e $5.45 \%$ em um cenário de trânsito fraco.

Por se tratar de tecnologias emergentes, alguns desafios ainda precisam ser contornados. O principal desafio provavelmente trata-se do padrão de mobilidade altamente dinâmico da rede veicular, o que torna difícil a disseminação de mensagens na rede e a manutenção dos agrupamentos formados pelos veículos. A adoção da comunicação D2D da quinta geração de telefonia celular irá impulsionar a capacidade de sensoriamento da rede, no qual diversos dispositivos com capacidade de sensoriamento poderão contribuir com novas informações. Entretanto, a heterogeneidade das fontes de informações constituem um desafio a ser enfrentado. Além disso, embora uma grande quantidade de dados proporcione uma melhor amostragem do ambiente sensoreado, a imposição de um sensoriamento em tempo real exige meios que ofereçam escalabilidade à rede.

Embora os experimentos tenham sido conduzidos utilizando um cenário altamente realista, apenas a simulação de mobilidade foi considerada neste estudo. Desta forma, um possível aprofundamento deste trabalho seria a inclusão da simulação de rede, em que os planos de controle e de dados da rede celular poderiam ser devidamente simulados. Fenômenos que poderiam ocasionar a degradação e perda do sinal também poderiam ser levados em consideração.

\section{Referências}

Bazzi, A., Masini, B. M., Zanella, A., and Pasolini, G. (2015). IEEE 802.11p for cellular offloading in vehicular sensor networks. COMPUTER COMMUNICATIONS, 60:97108.

Behrisch, M., Bieker, L., Erdmann, J., and Krajzewicz, D. (2011). Sumo - simulation of urban mobility. The Third International Conference on Advances in System Simulation, pages 55-60.

Campolo, C., Molinaro, A., and Scopigno, R. (2015). Vehicular ad hoc Networks: Standards, Solutions, and Research. Springer, 1 edition.

Costa, L. D., Rodrigues, F. a., Travieso, G., and Boas, P. R. V. (2007). Characterization of complex networks: a survey of measurements. Advances in Physics, 56(1):167-242.

Dua, A., Kumar, N., and Bawa, S. (2017). Game theoretic approach for real-time data dissemination and offloading in vehicular ad hoc networks. Journal of Real-Time Image Processing, 13(3):627-644.

Forecast, C. V. (2017). Global Mobile Data Traffic Forecast Update, 2016-2021. https://www.cisco.com/c/en/us/solutions/collateral/service-provider/visualnetworking-index-vni/mobile-white-paper-c11-520862.html. [Acesso em: 25 nov. 2018].

Freeman, L. C. (1978). Centrality in social networks conceptual clarification. Social Networks, 1(3):215 - 239. 
Hartenstein, H. and Laberteaux, K. (2010). VANET: Vehicular Applications and InterNetworking Technologies. Wiley.

Kolios, P., Ellinas, G., and Panayiotou, C. (2013). Vehicular Data Offloading under Uncertain Journey Planners. In 2013 INTERNATIONAL CONFERENCE ON CONNECTED VEHICLES AND EXPO (ICCVE), International Conference on Connected Vehicles and Expo, pages 544-550.

Lee, S. and Lee, S. (2013). User-centric offloading to WLAN in WLAN/3G vehicular networks. Wireless Personal Communications, 70(4):1925-1940.

Lee, U., Magistretti, E., Zhou, B., Gerla, M., Bellavista, P., and Corradi, A. (2006). Efficient data harvesting in mobile sensor platforms. In Fourth Annual IEEE International Conference on Pervasive Computing and Communications Workshops (PERCOMW'06), pages 5 pp.-356.

Li, Y., Jin, D., Wang, Z., Zeng, L., and Chen, S. (2014). Coding or Not: Optimal Mobile Data Offloading in Opportunistic Vehicular Networks. IEEE TRANSACTIONS ON INTELLIGENT TRANSPORTATION SYSTEMS, 15(1):318-333.

Li, Z., Liu, Y., Zhu, H., and Sun, L. (2015). Coff: Contact-duration-aware cellular traffic offloading over delay tolerant networks. IEEE Transactions on Vehicular Technology, 64(11):5257-5268.

Lin, Y.-D., Ku, C.-Y., Lai, Y.-C., and Liang, Y.-H. (2018). Wi-fi offloading between lte and wlan with combined ue and bs information. Wireless Networks, 24(4):1033-1042.

Mao, G., Zhang, Z., and Anderson, B. D. O. (2016). Cooperative Content Dissemination and Offloading in Heterogeneous Mobile Networks. IEEE TRANSACTIONS ON VEHICULAR TECHNOLOGY, 65(8):6573-6587.

Mezghani, F., Dhaou, R., Nogueira, M., and Beylot, A.-L. (2016). Offloading Cellular Networks Through V2V Communications - How to Select the Seed-Vehicles? In 2016 IEEE INTERNATIONAL CONFERENCE ON COMMUNICATIONS (ICC), IEEE International Conference on Communications. IEEE; IEEE Commun Soc.

Nunes, D. F., Moreira, E. S., Kimura, B. Y., Sastry, N., and Mahmoodi, T. (2017). Attraction-Area Based Geo-Clustering for LTE Vehicular CrowdSensing Data Offloading. Proceedings of the 20th ACM International Conference on Modelling, Analysis and Simulation of Wireless and Mobile Systems - MSWiM '17, pages 323-327.

Salvo, P., Turcanu, I., Cuomo, F., Baiocchi, A., and Rubin, I. (2016). LTE Floating Car Data application off-loading via VANET driven clustering formation. In $201612 \mathrm{TH}$ ANNUAL CONFERENCE ON WIRELESS ON-DEMAND NETWORK SYSTEMS AND SERVICES (WONS), pages 192-199.

Stanica, R., Fiore, M., and Malandrino, F. (2013). Offloading floating car data. 2013 IEEE 14th International Symposium on a World of Wireless, Mobile and Multimedia Networks, WoWMoM 2013, pages 1-9.

Uppoor, S., Trullols-Cruces, O., Fiore, M., and Barcelo-Ordinas, J. M. (2014). Generation and analysis of a large-scale urban vehicular mobility dataset. IEEE Transactions on Mobile Computing, 13(5):1061-1075. 
Wang, J., Jiang, C., Zhang, K., Quek, T. Q. S., Ren, Y., and Hanzo, L. (2018). Vehicular sensing networks in a smart city: Principles, technologies and applications. IEEE Wireless Communications, 25(1):122-132.

Yuan, Q., Li, J., Liu, Z., and Yang, F. (2016). Space and time constrained data offloading in vehicular networks. In 2016 IEEE 18th International Conference on High Performance Computing and Communications; IEEE 14th International Conference on Smart City; IEEE 2nd International Conference on Data Science and Systems (HPCC/SmartCity/DSS), pages 398-405.

Zhu, X., Li, Y., Jin, D., and Lu, J. (2017). Contact-Aware Optimal Resource Allocation for Mobile Data Offloading in Opportunistic Vehicular Networks. IEEE Transactions on Vehicular Technology, 66(8):7384-7399. 